\title{
Numerical Modeling of Melt Meniscus Shape in the Induction Crucible Furnace \\ by Using Open-Source Software
}

\author{
Maksim U. Khatsayuk and Nikita V. Sizganov* \\ Siberian Federal University \\ Krasnoyarsk, Russian Federation
}

Received 18.03.2020, received in revised form 30.03.2020, accepted 09.04.2020

Abstract. The article describes the definition of dependencies connecting the parameters of an induction crucible furnace and the shape of the meniscus using a mathematical model. The analysis of the data obtained and a qualitative assessment of the mathematical model based on the open non-commercial software Elmer and OpenFOAM are carried out. The created models were debugged for use in further studies of the free surface of the melt.

Keywords: numerical modeling, magnetohydrodynamics, free surface, induction crucible furnace, EOF-Library.

\section{Численное моделирование формы мениска расплава \\ в индукционной тигельной печи \\ с использованием открытого \\ программного обеспечения}

М.Ю. Хацаюк, Н.В. Сизганов

Сибирский федеральный университет Российская Федераиия, Красноярск

Аннотация. В статье рассмотрено определение зависимостей, связывающих параметры индукционной тигельной печи и форму мениска с помощью математической модели. Проведен

(C) Siberian Federal University. All rights reserved

This work is licensed under a Creative Commons Attribution-Non Commercial 4.0 International License (CC BY-NC 4.0).

* Corresponding author E-mail address: vitz1234@gmail.com 
анализ полученных данных и качественная оценка математической модели на базе открытого некоммерческого программного обеспечения Elmer и OpenFOAM. Выполнена отладка моделей, созданных для использования в дальнейших исследованиях свободной поверхности расплава.

Ключевые слова: численное моделирование, магнитная гидродинамика, свободная поверхность, индукционная тигельная печь, EOF-Library.

Цитирование: Хацаюк, М.Ю. Численное моделирование формы мениска расплава в индукционной тигельной печи с использованием открытого программного обеспечения / М.Ю. Хацаюк, Н.В. Сизганов // Журн. Сиб. федер. ун-та. Техника и технологии, 2020. 13(4). C. 487-494. DOI: 10.17516/1999-494X-0239

\section{Введение}

Расплавленный металл в электромагнитном поле индукционной тигельной печи (ИТП) под воздействием радиальной составляющей объемных сил Лоренца $f_{l}$ начинает движение, образующее мениск на свободной поверхности расплава (рис. 1). Формирующаяся естественная двухконтурная электродинамическая циркуляция расплава имеет как положительные - выравнивание температуры и химического состава, химическое взаимодействие металла со шлаком, - так и отрицательные свойства - прорыв окисной пленки, неэффективное использование шлаков, сравнительно слабое смешивание циркулирующих контуров и пр. [1-3].

Выделим уже известные зависимости для мениска в ИТП: высота мениска растет при увеличении удельной мощности; при неизменной мощности, передаваемой в расплав, силовое воздействие на него усиливается с понижением частоты [2]. Также известно, что выраженность мениска будет зависеть не только от электромагнитных (ЭМ), но и от геометрических параметров ЭМ-системы, а именно от положения торца катушки относительно поверхности расплава, от расстояния между расплавом и катушкой $[1,2]$.

Исследования мениска проводились с помощью аналитических методов [1, 2], одномерными численными моделями без учета гидродинамики [4], двухмерными осесимметричными численными моделями с учетом гидродинамики [5], а также с использованием современного

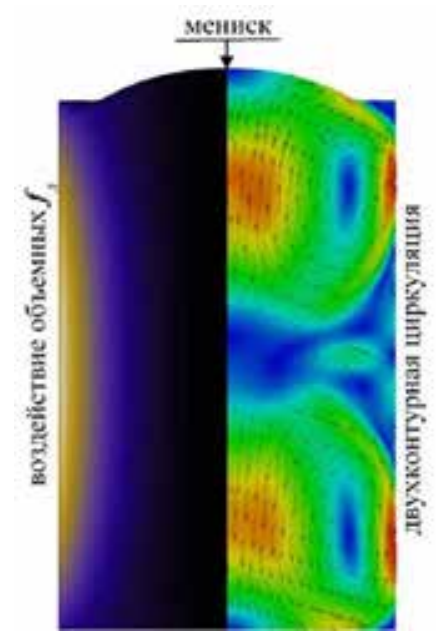

Рис. 1. Формирование мениска в тигле индукционной печи

Fig. 1. The formation of the meniscus in the crucible of an induction furnace

$$
-488-
$$


программного обеспечения с доработками для связывания ЭМ и гидродинамических задач: Ansys classic + CFX [6], Ansys classic + Ansys fluent [7], Elmer + OpenFOAM [8] и Comsol + Ansys fluent [9]. Свободная поверхность расплава в переменном электромагнитном поле является МГД-задачей, относящейся к разряду комплексных физических задач. Для решения такого типа задач предложено использование открытой библиотеки EOF-Library [10], поскольку она обладает рядом преимуществ перед остальными связками решателей: имеет открытый исходный код, распространяется бесплатно и позволяет выполнять связку двух решателей параллельно, без дополнительных программ, которые увеличивают время расчета.

Целью данной работы служит выявление основных закономерностей формирования мениска на свободной поверхности эвтектического сплава GaInSn в электромагнитном поле (ЭМП) ИТП. Теоретический эксперимент проводили на математической модели МГД-процессов, решаемой численным методом с помощью открытой библиотеки EOF-Library. Исследование зависимостей параметров мениска от энергетических и геометрических параметров позволило оценить возможности и выполнить отладку созданных моделей.

\section{Описание расчетной системы}

При исследованиях электромагнитных процессов в ИТП рассматривается система «индуктор - загрузка». Индуктор в зависимости от энергетических параметров системы может быть выполнен в виде многовитковой катушки переменного тока с естественным воздушным охлаждением или в виде массивной трубки с принудительным водяным охлаждением. Загрузкой в случае применения непроводящего тигля или тигля прозрачного для ЭМП на данных частотах является только расплав.

Для численного решения были приняты следующие допущения: витки реального индуктора заменяют одним витком с соответствующей магнитодвижущей силой (МДС); непроводящие и незначительные геометрические элементы конструкции не учитываются; задача решается в двухмерной осесимметричной постановке; поле в электромагнитной задаче изменяется по гармоническому закону; тепловыми процессами и естественным конвективным движением расплава пренебрегается. Допущения являются стандартными для данного класса задач и не приводят к значительному увеличению отклонения результатов, однако позволяют значительно сэкономить вычислительные ресурсы. Схематичное представление геометрии расчетной области с основными размерами показано на рис. 2, где 1 - расплав, 2 - индуктор. Размеры брали в масштабах физической модели индукционной тигельной печи для изучения МГД-явлений на низкотемпературном эвтектическом сплаве с металлической проводимостью.

Гидродинамическую задачу решали только в области расплава. Ожидаемое число Рейнольдса в расплаве $R e \approx 38000$, что много больше критического значения, и следует ожидать образование турбулентных течений. Их учет производили с помощью $k$ - $\varepsilon$-модели турбулентности. В условиях переменного магнитного поля в гидродинамической задаче учитывали силовое воздействие через дополнительный источник движения. Моделирование свободной поверхности реализовано с помощью метода объема жидкости $(V O F)$, где $\alpha$ характеризует объемную долю жидкости в ячейке сетки.

Электромагнитную задачу решали в квазистационарной постановке, с указанием эквивалентной плотности тока. Расчетная область, помимо индуктора и загрузки, заполнена возду-

$$
-489-
$$




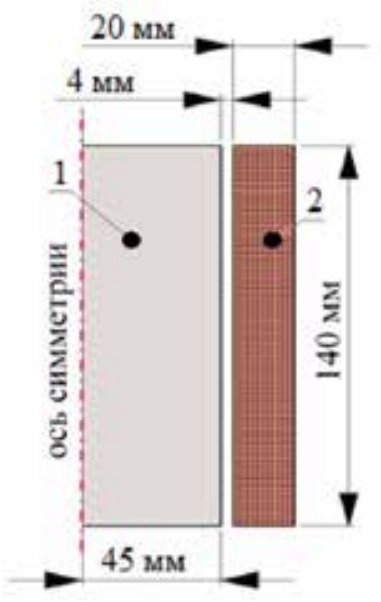

Рис. 2. Геометрия расчетной области

Fig. 2. Geometry of the computational domain

Таблица 1. Физические свойства материалов

Table 1. Physical properties of materials

\begin{tabular}{|c|c|c|c|c|}
\hline$\Pi / \Pi$ & Параметр & Обозначение & $\begin{array}{c}\text { Единицы } \\
\text { измерения }\end{array}$ & Величина \\
\hline \multicolumn{5}{|c|}{ Расплав GaInSn } \\
\hline 1 & Плотность & $\rho$ & $\kappa \Gamma / \mathrm{M}^{3}$ & 6080 \\
\hline 2 & Проводимость & $\sigma$ & См/м & $3.85 \times 10^{6}$ \\
\hline 3 & Кинематическая вязкость & $v$ & $\mathbf{M}^{2} / \mathrm{c}$ & $2.6315 \times 10^{-7}$ \\
\hline \multicolumn{5}{|c|}{ Медный индуктор } \\
\hline 4 & Относительная магнитная проницаемость & $\mu$ & o.e. & 1 \\
\hline 5 & Проводимость & $\sigma$ & $\mathrm{CM} / \mathrm{M}$ & $5.96 \times 10^{7}$ \\
\hline \multicolumn{5}{|c|}{ Окружающчая среда - воздух } \\
\hline 6 & Плотность & $\rho$ & $\kappa \Gamma / \mathrm{M}^{3}$ & 1 \\
\hline 7 & Кинематическая вязкость & $v$ & $\mathrm{M}^{2} / \mathrm{c}$ & $1.48 \times 10^{-5}$ \\
\hline 8 & Поверхностное натяжение расплав - воздух & $\gamma$ & $\mathrm{H} / \mathrm{M}$ & 0.72 \\
\hline
\end{tabular}

хом, границы которого в электромагнитной задаче удалены от индуктора для снижения влияния граничных условий.

Во время расчета МГД-задачи пересчет ЭМ-части инициировался в зависимости от изменения положения свободной поверхности, определяемой в ходе решения гидродинамической части. Такой подход позволяет сократить количество внутренних решений электромагнитной части, за счет чего сильно сокращается общий объем вычислений. Далее по тексту и на графиках для обозначения величины МДС используется полный ток I, A•вum. Все результаты исследования, связанные с границей раздела сред, будут показаны для $\alpha=0,5$. В результатах представлен установившийся режим нестационарного расчета указанной МГД-задачи. Свойства используемых материалов, необходимые для решения МГД-задачи, представлены в табл. 1.

$$
-490-
$$




\section{Исследование формы мениска в зависимости от энергетических и геометрических параметров}

Определение зависимостей, связывающих параметры ИТП и форму мениска, потребовало ввести следующие оценочные параметры: общая высота мениска $\Delta h=h_{\max }-h_{\min }$, где $h_{\max }, h_{\min }-$ максимальное и минимальное осевое положение мениска; отклонение мениска от положения равновесия $\Delta h_{0}=h_{\max }-h_{0}$, где $h_{0}$ - начальный уровень расплава (рис. $3 a$ ); $U_{c p},-$ средняя скорость циркуляции расплава.

Данные параметры позволили охарактеризовать наблюдаемые процессы в расплаве с точки зрения гидродинамики. По мере проведения численного эксперимента варьировались два ЭМ параметра системы - частота питания индуктора $f_{0}$ и полный ток $I$, а также наполненность тигля расплавом. Рассмотрим зависимость оценочных параметров от полного тока, представленную на рис. 36 .

На рис. $3 б$ наблюдается квадратичная зависимость высоты мениска $(\Delta h)$ от тока и, соответственно, линейная зависимость от мощности системы. Средняя же скорость $\left(U_{c p}\right)$ линейно связана с величиной тока.

На рис. 4 представлена зависимость оценочных параметров от относительной глубины проникновения $\Delta^{\prime}-1 /\left(R \sqrt{\pi f_{0} \sigma \mu_{0}}\right)$, где $R$ - радиус расплава, $\mu_{0}$ - магнитная постоянная; $\sigma$ - проводимость расплава; $f_{0}$ - частота питания индуктора.

Увеличение частоты питания индуктора (уменьшение $\Delta^{\prime}$ ) дает монотонный рост интегрального значения сил Лоренца $F_{Л}$, что отражается увеличением всех оценочных параметров. Когда глубина проникновения становится близкой к радиусу заготовки (для используемых в расчете размеров порядка 500 Гц), рост электромагнитного давления вызывает увеличение гидростатического давления для уравновешивания системы, что видно из отклонения мениска от положения равновесия $\left(\Delta h_{0}\right)$. Скорости расплава $\left(U_{c p}\right)$ зависят от величины $F_{\text {Л }}$ в то время как параметры свободной поверхности и силы Лоренца имеют более сложную взаимосвязь. Форма мениска зависит от величины, однако локальный характер распределения сил $F_{Л}$, а именно осевое распределение в зоне мениска из-за проявления краевого эффекта, имеет большее влияние на свободную поверхность, что характеризуется разными значениями $\Delta^{\prime}$ максимумов

a)

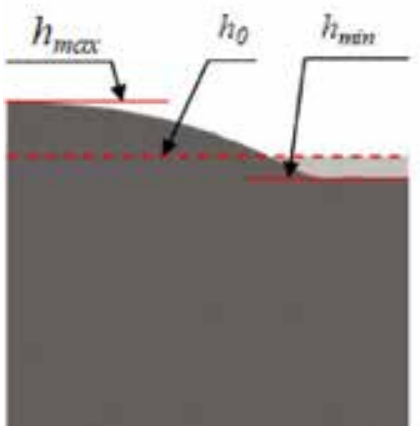

б)

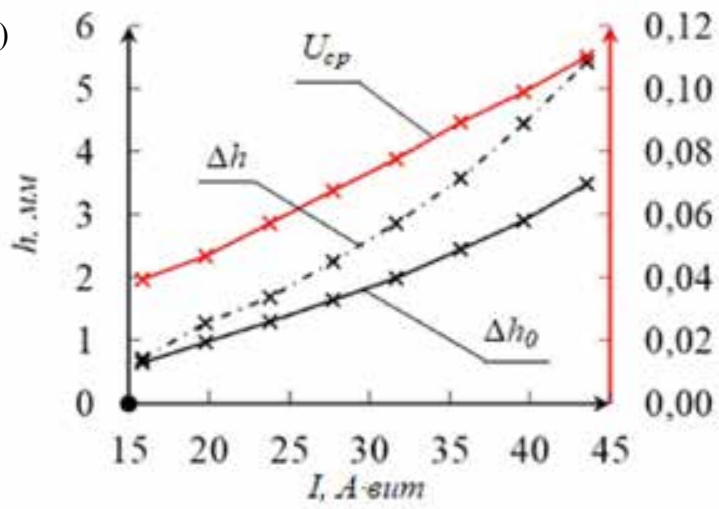

12

0,10

Рис. 3. Осевые положения мениска (a); зависимость оценочных параметров от полного тока (б)

Fig. 3. Axial position of the meniscus (a); dependence of estimated parameters on the total current $(\sigma)$ 


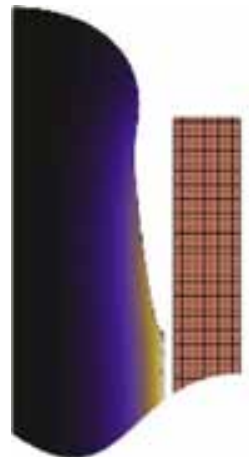

a)

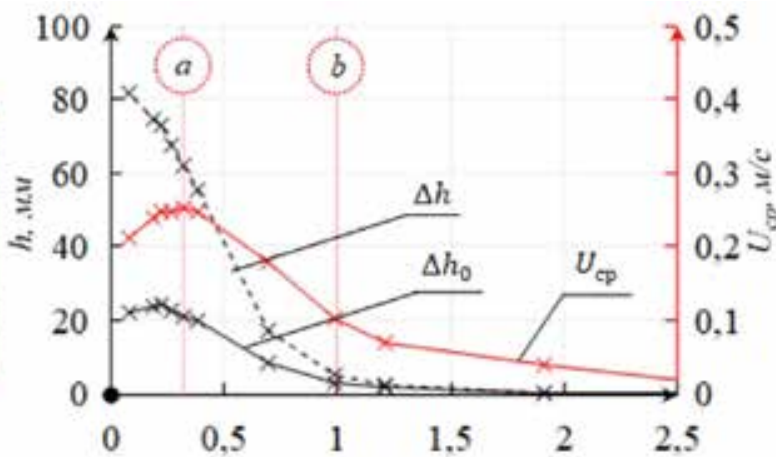

$A^{\prime}$, o.e.

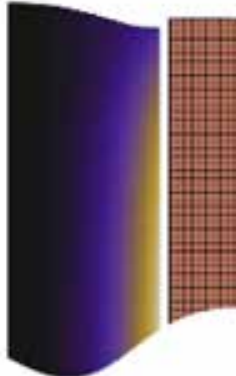

b)

Рис. 4. Зависимость оценочных параметров от относительной глубины проникновения

Fig. 4. The dependence of the estimated parameters on the relative depth of penetration

указанных параметров. Дальнейшее увеличение частоты приводит к снижению интегрального значения, но к увеличению плотности сил Лоренца, чем можно объяснить различие в характере поведения параметров $\Delta h_{0}$ и $\Delta h$ в данном диапазоне частот. Такой характер увеличения высоты мениска $(\Delta h)$ объясняется тем, что снижение точки мениска $h_{\min }$ связано с увеличением ЭМ-давления в пограничном слое на поверхность расплава вплоть до полного отрыва расплава от стенок тигля.

Для обозначения наполненности тигля расплавом относительно индуктора введен параметр $k_{m}=h_{0} / h_{i n d}$, где $h_{i n d}$ - высота индуктора, и выполнен анализ его влияния на характеристики мениска (рис. 5).

При опустошении тигля $k_{m}<1$ (рис. $5 a$ ) высота мениска будет увеличиваться, это объясняется возрастанием осевой составляющей силы Лоренца из-за краевых эффектов. Создаваемое дополнительное давление на свободную поверхность около точки контакта трех сред (воз-

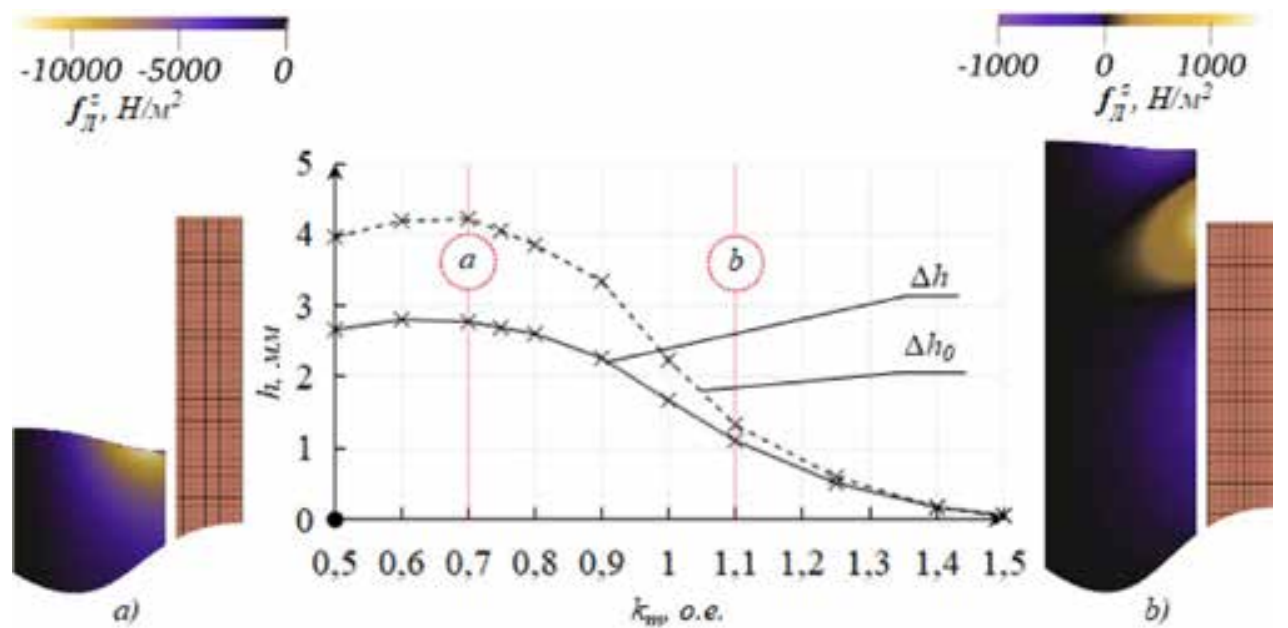

Рис. 5. Зависимость оценочных параметров от наполненности тигля расплавом

Fig. 5. The dependence of the estimated parameters on the filling of the crucible with the melt 
дух-расплав-тигель), усиливает верхний контур циркуляции и увеличивает высоту мениска. Дальнейшие опустошение тигля приводит к незначительному снижению высоты мениска из-за ограничения общего объема расплава для замыкания гидродинамических течений. При переполнении тигля $k_{m}>1$ (рис. $5 b$ ) исчезает осевая составляющая электромагнитных сил и вместе с ней мениск. Из [1] также известно, что в данной зоне возможно формирование вторичных течений над индуктором.

\section{Заключение}

Результаты нестационарного расчета созданных моделей после отладки коррелируют с верифицированными результатами, проведенными в авторитетных работах по данным направлениям, что говорит о достаточной достоверности модели при сохранении ее преимуществ перед остальными способами сопряженного численного решения МГД-задач.

Полученные результаты позволили выбрать базовые значения основных параметров системы для дальнейшего решения нестационарной задачи, направленной на изучение переходных и колебательных гидродинамических процессов.

Проведенный анализ выполненных исследований с учетом гидродинамики дополняет задачу свободной поверхности в электромагнитном поле. В частности, анализ положения катушки относительно поверхности расплава дает более полное представление о формировании мениска, что может быть использовано для более эффективного проведения плавки различных металлов и сплавов в холодном тигле с учетом технологических требований и ограничений. Также возникает возможность проведения исследований локального воздействия на свободную поверхность расплава с целью управления формой мениска и характером локальных течений, что позволит исключить отрицательные свойства связанных явлений в индукционной тигельной печи.

\section{Благодарность / Acknowledgement}

Исследование выполнено при финансовой поддержке РФФИ и Правительства Красноярского края в рамках научного проекта № 18-48-242013 «Исследование влияния пространственных и частотно-временных распределений электромагнитных сил в расплаве на характер протекания МГД-процессов в жидком металле».

No. 18-48-242013 «Study of the influence of spatial and frequency-time distributions of electromagnetic forces in the melt on the nature of the course of MHD processes in liquid metal.»

\section{Список литературы / References}

[1] Слухоцкий А.Е., Немков В.С., Павлов Н.А., Бамунэр А.В. Установки индукционного нагрева. Л.: Энергоиздат, 1981. 328 с. [Slukhotsky A.E., Nemkov V.S., Pavlov N.A., Bamuner A.V. Induction heating plants Induction heating plants, L., Energoizdat, 1981, 328 p. (in Russian)]

[2] Вайнберг А.М. Индукционные плавильные печи. М.: Государственное энергетическое издательство, 1960. 456 с. [Veinberg A.M. Induction melting furnaces, M., State Energy Publishing House, 1960, 456 p. (in Russian)]

[3] Baake E., Langejuergen M., Kirpo M., Jakovics A. Analysis of transient heat and mass transfer processes in the melt of induction channel furnaces using LES, Magnetohydrodynamics, 2009, 3, 385-392.

$$
-493-
$$


[4] Nakata H., Etay J. Meniscus shape of molten steel under alternating magnetic field. ISIJ Internationai, 1992, 32(4), 521-528.

[5] Sakane J., Li B., Evans J. Mathematical modeling of meniscus profile and melt flow in electromagnetic casters. Metall. Mater. Trans. B, 19 (3) (1988), 397-408.

[6] Spitans S., Jakovics A., Baake E., Nacke B. Numerical modelling of free surface dynamics of conductive melt in the induction crucible furnace. Magnetohydrodynamics, 2010, 46(4), 317-328.

[7] Хацаюк М.Ю. Теория и моделирование магнитогидродинамических процессов в электротехнологических комплексах металлургического назначения, дис. ... д-ра техн. наук. СанктПетербург, 2020, 338 c. [Khatsayuk M.U. Theory and modeling of magnetohydrodynamics processes in elctrotechnological complexes of metallurgical purpose, diss. ... dr. of tech. sci. St. Petersburg, 2020, 338 p. (in Russian)]

[8] Vencels J., Jakovics A., Geza V., Scepanskis M. EOF Library: open-source Elmer and OpenFOAM coupler for simulation of MHD with free surface. [Electronic resource] - Access: https://www.researchgate.net/publication/318418395_EOF_Library_Open-Source_Elmer_and_ OpenFOAM_Coupler_for_Simulation_of_MHD_With_Free_Surface

[9] Bourrou R., Budenkova O., Charvin P., Lafon C., Lemont F., Gagnoud A. Numerical study of the hydrodynamics in a two-phase induction melter for nuclear waste treatment under various operating parameters. European Journal of Mechanics/B Fluids, 2020, 79, 181-189.

[10]Vencels J., Råback P., Geža V. EOF-Library: Open-source Elmer FEM and OpenFOAM coupler for electromagnetics and fluid dynamics. SoftwareX, 2019, 9, 68-72. 